\title{
Investigating the Multi-Target Pharmacological Mechanism of Rhodiola Wallichiana Var. Cholaensis Acting on Angina Pectoris by Combined Network Pharmacology and Molecular Docking
}

\section{Haitao Zhang}

Fujian Provincial Maternity and Children's Hospital https://orcid.org/0000-0001-6417-4622

\section{Xudong Zhuang}

Fujian Province Hospital for Women and Children: Fujian Provincial Maternity and Children's Hospital

\section{Zhixiong Li}

Fujian Provincial Maternity and Children's Hospital: Fujian Provincial Maternity and Children's Hospital Xinrui Wang ( $\square$ wanxiru@sjtu.edu.cn)

Affiliated Hospital of Fujian Medical University

\section{Research}

Keywords: Rhodiola wallichiana var. cholaensis, Network pharmacology, angina pectoris, Signaling pathway, Molecular docking

Posted Date: September 21st, 2021

DOI: https://doi.org/10.21203/rs.3.rs-892492/v1

License: (a) (i) This work is licensed under a Creative Commons Attribution 4.0 International License.

Read Full License 


\section{Abstract}

Background: Angina pectoris is a kind of cardiovascular disease, which is caused by epicardial stenosis, microvascular dysfunction, dynamic stenosis area contraction or more comprehensive factors, eventually leading to Coronary artery disease. Rhodiola wallichiana var. cholaensis, which is one of the most wellknown Traditional Chinese Medicine, is widely used to treat angina pectoris. However, the possible underlying mechanisms remain to be elucidated.

Methods: In this study, systematic and comprehensive network pharmacology and molecular docking were utilized for the first time to reveal the potential pharmacological mechanisms of RW on AP. Firstly, the relative compounds were obtained by mining literature and potential targets of these compounds by target predicting were collected. Then, We built the AP target database using the DigSee and GeneCards Database. Based on the data, GO and KEGG pathway enrichment analysis, protein-protein interaction (PPI) analysis were performed and screen the hub targets by topology. Furthermore, evaluation of the binding potential of key targets and compounds through molecular docking.

Results: The results indicate that 218 known RW therapeutic targets were selected. By systematic analysis, identified 9 hub targets (VEGFA, GAPDH, TP53, AKT1, CASP3, STAT3, TNF, MAPK1 and JUN) mainly involved in the complex treating effects associated with the protection of vascular endothelium, as well as the regulation of glucose metabolism, cellular processes, inflammatory responses, and cellular signal transduction.

Conclusion: The results of this study preliminarily explain the potential targets and signaling pathways of RW in AP therapy and lay a good foundation for further experimental studies and clinical trials.

\section{Background}

Angina pectoris (AP) is a kind of cardiovascular disease, which is caused by epicardial stenosis, microvascular dysfunction, dynamic stenosis area contraction or more comprehensive factors, eventually leading to Coronary artery disease (CAD)[1]. It is well known that myocardial ischemia is a typical symptom of AP. Therefore, conventional medicines for the treatment of AP, such as nitrates, beta-blockers, calcium antagonists, aspirin, and ACE inhibitors, are mainly used to alleviate the symptoms of myocardial ischemic and then improve AP prognosis. However, all the drugs mentioned above may cause some adverse reactions. For example, misuse of nitrates usually increase the risk of drug resistance and a rebound of symptoms, which could lead to serious adverse reactions such as headaches and flushing[2].

Traditional Chinese Medicine (TCM) is an integrated medical system with multi-components and multitargets, which has thousands of years history. It has potential application value in clinical practice in China[3]. The Rhodiola wallichiana var. cholaensis (RW) is a common species of the genus Rhodiola which is one of the most popular medicinal plants in Asia[4]. Generally, RW has widely been used for the treatment of cardiovascular diseases, diarrhea, hysteria, hernias, headaches as well as cognitive 
dysfunction[5]. It has also been reported that RW possesses a beneficial effect on kidney stones, swellings, back pain, as well as mood disorders[6, 7]. Additionally, RW possesses a wide range of pharmacological activities such as antiaging, anti-oxidative, anti-inflammatory, anti-cancer, anti-fatigue and neuroprotective effects [8], contributing to the presence of various phytochemicals such as phenols and flavonoids[9-13]. For example, Phenylethanoid derivatives such as Salidroside, Rosavins, Rosin, and P-tyrosol are responsible for the treatment of depression, fatigue, and cognitive dysfunction[5]. However, due to the multi-compound system of Traditional Chinese Medicine (TCM), the material basis and molecular mechanisms involved in RW remain unclear. Therefore, the development of modern and technologic approaches are urgently needed for the analysis of the action mechanisms of RW in the treatment of various diseases.

Network pharmacology is a new research method integrating pharmacodynamics, pharmacokinetics and network analysis in recent years, which mainly elucidates the possible mechanism of TCM prescription in the treatment of various diseases from the perspective of proteomics systems[14-16].In particular, it has been a novel research strategy to expound the interaction relationship of TCM in multi-component and multi-target, as well as to study the mechanism of multi-target compounds affecting the biological network of TCM[17-19]. Hence, in this study, network pharmacology strategies, including drug similarity assessment, oral bioavailability prediction, multiple drug target prediction, and other network pharmacology techniques were used to study the potential role of RW against unstable AP mechanisms. The gene target network of RW was analyzed through network pharmacology, starting with the identification of active substances and key target genes whose expression is altered in AP and whose protein products are predicted to interact with active compounds in RW.The flow diagram of the pharmacological mechanism of RW treating AP is shown in Fig.1.

\section{Results}

\section{Targets Screening of RW and AP}

A total of 83 chemical ingredients were obtained from the RW according to related literature studies. After eliminating redundancy, there were 26 chemical ingredients (Table 1) and 671 corresponding targets of RW were acquired, as well as 1297 therapeutic targets for AP were obtained used by GeneCards database and DigSee database (Fig. 2B). Then the ultimate gene targets of RW acting on AP were obtained through mapping these targets to the components of the disease targets. As shown in Fig. 2C, 218 target genes corresponding RW candidate compounds of AP were gained for further research.

\section{Network Construction and Result Analysis}

It is well known that TCM works primarily through synergy between compounds and their targets. To elucidate the potential mechanism of this synergistic effect of RW on AP, it is necessary to understand the effect of each component in RW on its target protein. By analyzing the network of compounds and putative targets in the RW, as shown in Fig. 2A, our study shows that the network consists of 1754 edges and 697 nodes, of which 26 are component nodes and 671 are target nodes. There is no doubt that target 
genes with high degree and mediated centrality are most important in the antianginal role of RW in the network.

\section{Construction and Analysis of PPI Network}

To further investigate the potential mechanism of action of RW processing of APs, we submitted target genes acting on the corresponding components to the string database, constructed the following PPI network, and then screened highly reliable target protein interaction data with scoring $>0.7$. As individual proteins are unlikely to perform specific functions, proteins often interact to form large molecular complexes to perform their biological functions. At the same time, exploring and constructing PPI networks is an important aspect of understanding cell biological processes and biological functions. As shown in Fig. 3, the obtained PPI network files were imported into Cytoscape software, and after

adjusting the parameters, 70 key genes were selected according to their extent, which are associated with various pathogenic processes of AP.

\section{GO Functional Analysis}

The DAVID panel analyzed a total of 70 potential genes associated with AP. We performed GO analysis on the 70 targets to explore their general functions. As shown in Fig. 4 (A-C), the results of $\mathrm{GO}$ analysis showed that RW acted on key predicted targets of AP, and $20 \mathrm{GO}$ items were enriched with higher enrichment targets and lower p-values. Our results show that these predicted targets are mainly involved in the positive regulation of cell signaling, protein autophosphorylation and cell proliferation, suggesting that RW may regulate the biological function of AP through these pathways, thus playing a key role in antianginal pain.

\section{Kyoto Encyclopedia of Genes and Genomes (KEGG) Signaling Pathway Analysis}

To identify the relevant signaling pathways involved in the antianginal effect of RW, DAVID analysis was performed. A total of 34 KEGG signaling pathways was obtained, and 27 pathways were associated with AP. To show the results of the signaling pathways intuitively and explicitly, a bubble diagram was utilized (Fig. 4D). The bubble scale represents the number of genes; the depth of bubble color represents P-value. The change of color from green to red represents the P-value from low to high, and the size of the nodes indicates how many target genes are associated.

The KEGG analysis results indicated that the multiple channels and mechanisms of action of RW against AP.

The top 20 pathways with lower $P$ values and more genes enrichment are listed in (Fig. 4D), including PI3K-Akt signaling pathway, HIF-1 signaling pathway, Ras signaling pathway, thyroid hormone signaling pathway, and Toll-like receptor signaling pathway being enriched. These signaling pathways are closely related to the anti-AP effects of RW. To elucidate their interactions, we established a graphical network 
containing the main chemicals-targets-signaling pathway of RW (Fig. 5). The HIF-1 signaling pathway (hsa04066) with the smaller p-value will be analyzed as an important pathway (Fig. 6).

\section{Molecular docking simulation}

In this paper, nine potential targets with thirteen corresponding compounds were simulated by molecular docking, and the docking results were analyzed. Analysis shows higher-affinity results for compound and hub targets (Table 2). Using PyMOL software, these 13 compounds were observed to enter the active pocket of the protein. Taking the HJT8 (Salidroside) as an example for analysis. (Fig. 7) Salidroside small molecule forms 4 hydrogen bonds with Lys52, Asn152, Ser151 and Met106 residues and higher-affinity (Affinity $=-7.5 \mathrm{kcal} / \mathrm{mol}$ ) with MAPK1.

\section{Discussion}

TCM is a complex, multi-component, multi-target hybrid system that has long been used for the prevention and treatment of various cardiovascular diseases (CVDs)[20-22]. RW can effectively treat AP, but its pharmacological mechanism of action is still unclear. Therefore, in this study, we used a pharmacological network approach to identify the bioactive compounds, potential targets, and pathways regulated by these compounds for $\mathrm{RW}$ treatment of $\mathrm{AP}$.

Nine potential hub targets were identified based on selection and network topology analysis, including VEGFA, GAPDH, TP53, AKT1, CASP3, STAT3, TNF, MAPK1 and Jun. Numerous studies have shown that these targets above mentioned mainly involved in the protection of vascular endothelium, as well as the regulation of glucose metabolism, cellular processes, inflammatory responses, and cellular signal transduction. VEGF, a strong pro-angiogenesis cytokine, is secreted by vascular endothelial cells, which could increase the permeability of microvessels and venules, promote angiogenesis, thus improving myocardial hypoxia and relieving AP pectoris[23-25]. Recent studies have demonstrated that the inflammatory response is related to the occurrence of cardiovascular diseases such as coronary heart disease and AP, which may cause local endothelial activation, atherosclerotic plaque rupture, and then thrombosis form or rupture, leading to AP and myocardial infarction[26]. It is well-known that TNF-a, usually appearing in the early stage of inflammatory response, plays an important role in cell function regulation, immunity and inflammatory response, which could regulate atherosclerotic plaques and coronary heart disease by affecting vascular endothelial function and vascular remodeling[27]. Biasucci et al., Huang et al. studies have shown that proinflammatory cytokines were significantly increased in patients with AP compared to healthy individuals. However, it is important to note that this increased inflammatory activity may be related to the pathogenesis of AP. For example, TNF- $a$ may increase the expression of monocyte/macrophage tissue factors, as well as cell apoptosis by improving thrombotic activity, leading to the increase of matrix metalloproteinases in atherosclerotic plaques[2832]. AKT1 is an important protein in the PI3K pathway, which could regulate cell apoptosis, proliferation and antioxidant[33,34]. Han et al.[35] demonstrated that hypericin can reduce the inflammatory response by activating phosphorylated AKT and reducing TNF- $a$ and IL- 6 activity, thus alleviating myocardial ischemia-reperfusion injury. The p53 gene is an important apoptosis-related gene, 
divided into two types: wild type (wp53) and mutant type (mp53). The mutant p53 gene can promote cell growth and participate in the occurrence of various tumors. The main function of the wild-type p53 gene is to participate in the negative regulation of cell growth, limiting cell growth and division. In recent years, studies have found that the p53 gene is not only related to the occurrence and development of many tumors but also participates in the occurrence of apoptosis in the cardiovascular system[36-38]. C-JUN is the heterodimer form of activating protein 1 (AP-1). Previous studies have demonstrated that CJUN can induce the production of adhesion factors in endothelial cells, increase the expression of chemokines and the formation of foam cells, thus promoting the formation and development of atherosclerosis[39]. MAPK1 belongs to the Ser-Thr kinase protein family and has been previously reported in different features of cardiac modeling and regulation of inflammation, cell proliferation and differentiation[40, 41]. The high activity of Erk1/2 has been observed in T-lymphocytes from CAD patients, including ST-elevation myocardial infarction (STEMI), Non-STEMI and unstable AP[42]. A previous study demonstrated the EGFR-mediated cross-talk between MAPK and Akt1 signaling, which combined had an important role in abnormal vascular remodeling[43]. STAT3 is a latent transcription factor, initially identified as a cytokine signaling transductor[44],and is involved in a variety of biological processes such as cell proliferation[45], differentiation[46], and survival[47].

To understand the potential biological mechanism of RW against AP, GO and KEGG functional enrichment analysis of DAVID and KEGG were applied. Through the KEGG pathway analysis ( $\mathrm{p}$-value < 0.05), we recognized 21 AP-related signaling pathways, HIF-1, PI3K-Akt, MAPK, FoXO, TNF, Ras and tolllike receptor signaling pathway and so on. Accordingly, these pathways may be involved in the progress of AP. Based on P-value, we choose HIF-1 signaling pathway as most candidate signals for further study. $\mathrm{HIF}$ is a transcriptional complex that responds to changes in oxygen and provides a master regulator for cells to coordinate changes in gene transcription. HIF acts on all mammalian cell types and is ancient during evolution. At the molecular level, the HIF complex contains an alpha subunit and a beta subunit, both of which can be selected from several options. HIF- $\beta$ subunits are composed and also participate in heterogeneous reactions. The alpha subunit is regulated and is unique to hypoxic reactions. Under hypoxic conditions, HIF-1 $a$ is induced and highly expressed, transferring from the cytoplasm to the nucleus and initiating downstream gene expression, such as erythropoietin and VEGF. HIF-1 a could increase myocardial glucose intake and transportation to continuously provide the compensatory energy supply by regulating myocardial GLUT4 and PKM2 gene expression[48]. HIF-1 $a$ also facilitates the activation of PDK1 and PDK4 as well as UCP2 to enhance mitochondrial oxidative phosphorylation[49]. Moreover, NRF1 and TFAM play distinct roles in mitochondrial biogenesis[50] and the upregulation of NRF1 and TFAM promotes mitochondrial DNA synthesis in infarcted cardiac muscle[51]. Therefore, HIF-1 signaling pathway is activated in cardiomyocytes to produce continuous ATP in adaption to hypoxia, by shifting myocardial metabolism substrate to glucose intake and transportation[52].

In this study, GO enrichment analysis was adopted. The targets were connected with regulation of protein phosphorylation, nitric oxide biosynthetic, cell membrane region, platelet alpha granule, protein kinase and protein phosphatase et al. Therefore, the results suggest that RW treats AP by participating in these $\mathrm{BP}, \mathrm{CC}$ and MF. 
Molecular docking simulation analysis provides a visual interpretation of the interactions between key compounds and potential protein targets. For example, the Salidroside small molecule forms 4 hydrogen bonds primarily with Lys52, Asn152, Ser151 and Met106 residues on MAPK1. Salidroside has various pharmacological activities such as anti-fatigue, anti-oxidation, immune regulation and free radical scavenging. In recent years, in vivo and in vitro experiments have proved that the compound has positive effects on anti-cancer, anti-inflammatory, anti-oxidation, neuroprotection, myocardial protection, liver protection, kidney protection. It has extremely important application value in military, aerospace, sports and health medicine[53-56]. In addition, it is reported that salidroside can inhibit the release of LDH, CK and AST of human cardiomyocytes by increasing the expression of HIF- $1 a$, increase the content of SOD, and at the same time increase the activity of human cardiomyocytes and reduce the death and apoptosis of cells[57]. Taken together, we speculate that the major components of RW may play an important role in the treatment of AP through hub targets in these top signaling pathways. However, there are some limitations to our study. For example, these results are only based on screening of known RW chemical components, associated targets and signaling pathways from the literature and existing databases. Therefore, more in-depth studies are needed to characterize the underlying mechanisms.

\section{Conclusion}

The 26 components of RW and the presumed known therapeutic targets were collected by network pharmacology and molecular docking virtual computational methods to explore the potential mechanism of RW for AP treatment. As a result of the GO and KEGG pathway enrichment analysis, we found that these central targets improve angina by being involved in protecting vascular endothelium, regulating glucose metabolism, modulating cellular processes, modulating inflammatory responses, and participating in cell signaling. In summary, the results of this study initially predicted the potential mechanisms associated with RW against AP and demonstrated the characteristics of multi-target synergy. However, in future studies, animal experiments, molecular biology experiments and clinical studies are still needed to verify the mechanism of RW's effect on AP.

\section{Methods}

\section{Chemical Ingredients Database Building}

A total of 83 RW compounds were collected through literature mining. then their biomolecular activities were viewed using the PubChem database (https://pubchem.ncbi.nlm.nih.gov)[58]. Finally, the standard simplified molecular-input Standard Delay Format (SDF) and structural information of 42 compounds were obtained, including salidroside, tyrosol, quercetin and so on[59-61].

\section{Screening of Active Compounds of RW}

Based on the Swiss ADME ( http://www.swissadme.ch ) platform to predict the relevant parameters of the absorption and drug-like properties of candidate compounds, upload the SDF format file under the item " Chemical Ingredients Database Building" to the Swiss ADME platform. First, set the gastrointestinal 
absorption as "High" results as the conditions under which the drug can be absorbed, used to screen for active compounds with good oral bioavailability; second, set 5 types of drug predictability (Lipinski, Ghose, Veber, Egan, Muegge) and there are 3 or more compounds that are "Yes" in the results, which can be used as active compounds.

\section{Active compound target prediction}

Import the SDF format file into the Swiss Target Prediction (http://www.Swisstargetprediction.ch/) and PharmMapper (http://www.lilab-ecust.cn/pharmmapper/) platform, set the property to "Homo sapiens", collect all the predicted targets, and de-weight the targets.

\section{The Prediction of Known Therapeutic Targets}

Genes associated with AP were collected and screened from the GeneCards database (http://www.genecards.org/), and DigSee database (http://210.107.182.61/geneSearch/). We searched GeneCards and DigSee using the keywords "angina pectoris" with the species limited as "Homo sapiens". Then the top 1000 target genes were selected from the GeneCards database, and 541 targets were gained from the DigSee database. Finally, 1297 genes were collected when removing duplicates. To obtain the potential target genes of RW that played a major role in AP, the AP-related genes were compared with potential gene targets of active components.

\section{Network Construction}

A network analysis was performed to scientifically explain the complex relationship between AP-related compounds and targets. Subsequently, based on the protein-protein interactions (PPI), we linked the putative targets of RW, the AP-related targets, and interactional proteins together. Then, to illustrate the relationship between the possible targets of RW and known targets of AP, the drug-compound-targetdisease network was constructed and visualized using the Cytoscape software (version 3.7.2, Boston, MA, USA). The PPI network of interaction between RW and AP was obtained via string software (http://string-db.org/cgi/input.pl), which the limiting conditions were "Homo sapiens" and a confidence score $\geq 0.4$, and the results were saved in TSV format and imported into the Cytoscape software to visualize and analyze the interaction network. We use the generated style from the statistics tool in Cytoscape to set the node size and color settings to reflect the size of the degree, and the thickness of the edge to reflect the size of the comprehensive score, to obtain the final protein interaction network. Degree refers to the number of links to node I, usually used to describe the topological importance of proteins in the network. Therefore, we use cytoHubba to select the central gene with the degree value as the tangent point to analyze the pharmacological effects of key targets.

\section{GO and KEGG pathway enrichment}

GO and KEGG pathway enrichment analysis were executed on the candidate targets using DAVID 6.8. GO gene enrichment analysis included three aspects: biological processes (BP), molecular functions (MF), and cell components (CC). With $\mathrm{P} \leq 0.05$ as the truncated value, the results were calculated by bilateral hypergeometric tests, including the identification of the enriched GO terms and the localization of the 
biological and molecular functions of these proteins. Finally, the bubble chart was plotted using the ImageGP platform (http://www.ehbio.com/ImageGP/index.php/Home/Index/index.html).

\section{Molecular docking simulation}

Core targets were obtained from the PPI network for molecular docking. Initially, we used AutoDockTools 1.5.6 to set the number of rotatable bonds for the 12 small molecule compounds. Subsequently, protein conformations were collected in the Protein Database (PDB, https://www.rcsb.org/). The screening conditions were set as follows: (1) the protein structure was obtained by $x$-crystal diffraction; (2) the organism comes from Homo sapiens; (3) Association action models were constructed by STRING and PDB database. Based on the above conditions, a total of 11 core target protein PDB IDs were collected.Meanwhile, PyMOL 2.7 (https://pymol.org/2/) and AutoDockTools were applied to add hydrogen and charge in addition to water molecules and pre-ligand small molecules. Finally, molecular docking calculations were performed using AutoDock Vina 1.1.2. The docking results were visualized using PyMOL and LigPlot ${ }^{+}$software.

\section{Abbreviations}

RW: Rhodiola wallichiana var. cholaensis; AP: Angina pectoris; PPI: protein-protein interaction; CAD: Coronary artery disease; TCM: Traditional Chinese Medicine; CVDs:Cardiovascular Diseases; SDF :Standard Delay Format; BP: biological processes, MF: molecular functions, CC: cell components .

\section{Declarations}

\section{Ethical Approval and Consent to participate}

Not applicable.

\section{Consent for publication}

Not applicable.

\section{Availability of data and materials}

All data generated or analysed during this study are included in this published article.

\section{Competing interests}

The authors declare that they have no competing interests. 


\section{Funding}

This work was supported by The central government guides local science and technology development projects [No. 2019L3019] and Startup Fund for scientific research, Fujian Medical University [No. 2020QH1203].

\section{Authors' contributions}

HTZ: Software, Validation, Visualization, Writing-original draft. XDZ: Writing-review \& editing. ZXL: Supervision. XRW: Conceptualization, Funding acquisition, Data curation, Formal analysis, Methodology

\section{Acknowledgements}

Thank you to Key Laboratory of Technical Evaluation of Fertility Regulation for Non-human Primate, National Health Commission, Fujian Maternity and Child Health Hospital, Affiliated Hospital of Fujian Medical University for the support of this research.

\section{Authors' information}

${ }^{1}$ Fujian Children's Hospital; Fuzhou China; ${ }^{2}$ Key Laboratory of Technical Evaluation of Fertility Regulation for Non-human Primate, National Health Commission, Fujian Maternity and Child Health Hospital, Affiliated Hospital of Fujian Medical University; Fuzhou, China; ${ }^{3}$ Medical Research Center, Fujian Maternity and Child Health Hospital; Fuzhou, China; ${ }^{4}$ Fujian Key Laboratory of Women and Children's Critical Diseases Research, Fujian Maternity and Child Health Hospital; Fuzhou, China;

\section{Authors' contributions}

HTZ: Software, Validation, Visualization, Writing-original draft. XDZ:Writing-review \& editing. ZXL: Supervision. XRW: Conceptualization, Funding acquisition, Data curation, Formal analysis, Methodology

\section{References}

1. Kloner RA, Chaitman B. Angina and Its Management. J Cardiovasc Pharmacol Ther. 2017;22:199209.

2. Thadani U. Nitrate tolerance, rebound, and their clinical relevance in stable angina pectoris, unstable angina, and heart failure. Cardiovasc Drugs Ther. 1997;10:735-42.

3. Yang Y, Li Y, Wang J, Sun K, Tao W, Wang Z, et al. Systematic Investigation of Ginkgo Biloba Leaves for Treating Cardio-cerebrovascular Diseases in an Animal Model. ACS Chem Biol. 2017;12:1363-72. 
4. van Diermen D, Marston A, Bravo J, Reist M, Carrupt PA, Hostettmann K. Monoamine oxidase inhibition by Rhodiola rosea L. roots. J Ethnopharmacol. 2009;122:397-401.

5. Nabavi SF, Braidy N, Orhan IE, Badiee A, Daglia M, Nabavi SM. Rhodiola rosea L. and Alzheimer's Disease: From Farm to Pharmacy. Phytother Res. 2016;30:532-9.

6. Panossian A, Wikman G, Sarris J. Rosenroot (Rhodiola rosea): traditional use, chemical composition, pharmacology and clinical efficacy. Phytomedicine. 2010;17:481-93.

7. Cropley M, Banks AP, Boyle J. The Effects of Rhodiola rosea L. Extract on Anxiety, Stress, Cognition and Other Mood Symptoms. Phytother Res. 2015;29:1934-9.

8. Ishaque S, Shamseer L, Bukutu C, Vohra S. Rhodiola rosea for physical and mental fatigue: a systematic review. BMC Complement Altern Med. 2012;12:70.

9. Zhang L, Yu H, Sun Y, Lin X, Chen B, Tan C, et al. Protective effects of salidroside on hydrogen peroxide-induced apoptosis in SH-SY5Y human neuroblastoma cells. Eur J Pharmacol. 2007;564:18-25.

10. Hu X, Lin S, Yu D, Qiu S, Zhang X, Mei R. A preliminary study: the anti-proliferation effect of salidroside on different human cancer cell lines. Cell Biol Toxicol. 2010;26:499-507.

11. Hu X, Zhang X, Qiu S, Yu D, Lin S. Salidroside induces cell-cycle arrest and apoptosis in human breast cancer cells. Biochem Biophys Res Commun. 2010;398:62-7.

12. Mao GX, Deng HB, Yuan LG, Li DD, Li YY, Wang Z. Protective role of salidroside against aging in a mouse model induced by D-galactose. Biomed Environ Sci. 2010;23:161-6.

13. Chang X, Zhang K, Zhou R, Luo F, Zhu L, Gao J, et al. Cardioprotective effects of salidroside on myocardial ischemia-reperfusion injury in coronary artery occlusion-induced rats and Langendorffperfused rat hearts. Int J Cardiol. 2016;215:532-44.

14. Li X, Wu L, Liu W, Jin Y, Chen Q, Wang L, et al. A network pharmacology study of Chinese medicine QiShenYiQi to reveal its underlying multi-compound, multi-target, multi-pathway mode of action. PLoS One. 2014;9:e95004.

15. Sheng S, Wang J, Wang L, Liu H, Li P, Liu M, et al. Network pharmacology analyses of the antithrombotic pharmacological mechanism of Fufang Xueshuantong Capsule with experimental support using disseminated intravascular coagulation rats. J Ethnopharmacol. 2014;154:735-44.

16. Xu T, Li S, Sun Y, Pi Z, Liu S, Song F, et al. Systematically characterize the absorbed effective substances of Wutou Decoction and their metabolic pathways in rat plasma using UHPLC-Q-TOF-MS combined with a target network pharmacological analysis. J Pharm Biomed Anal. 2017;141:95-107.

17. Li X, Xu X, Wang J, Yu H, Wang X, Yang H, et al. A system-level investigation into the mechanisms of Chinese Traditional Medicine: Compound Danshen Formula for cardiovascular disease treatment. PLoS One. 2012;7:e43918.

18. Chandran U, Patwardhan B. Network ethnopharmacological evaluation of the immunomodulatory activity of Withania somnifera. J Ethnopharmacol. 2017;197:250-6. 
19. Kim BY, Song KH, Lim CY, Cho SI. Therapeutic properties of Scutellaria baicalensis in $\mathrm{db} / \mathrm{db} \mathrm{mice}$ evaluated using Connectivity Map and network pharmacology. Sci Rep. 2017;7:41711.

20. Hao P, Jiang F, Cheng J, Ma L, Zhang Y, Zhao Y. Traditional Chinese Medicine for Cardiovascular Disease: Evidence and Potential Mechanisms. J Am Coll Cardiol. 2017;69:2952-66.

21. Hao DC, Xiao PG. Impact of Drug Metabolism/Pharmacokinetics and their Relevance Upon Traditional Medicine-based Cardiovascular Drug Research. Curr Drug Metab. 2019;20:556-74.

22. Shu Z, Wu T, Shahen M, Guo Z, Shu J, Wang HE, et al. System-Pharmacology Dissection of Traditional Chinese herbs SINI Decoction for Treatment of Cardiovascular Diseases. An Acad Bras Cienc. 2019;91:e20180424.

23. Waltenberger J, Kranz A, Beyer M. Neovascularization in the human heart is associated with expression of VEGF-A and its receptors Flt-1 (VEGFR-1) and KDR (VEGFR-2). Results from cardiomyopexy in ischemic cardiomyopathy. Angiogenesis. 1999;3:345-51.

24. Shibuya M. VEGF-VEGFR System as a Target for Suppressing Inflammation and other Diseases. Endocr Metab Immune Disord Drug Targets. 2015;15:135-44.

25. Lihong LGWLL. Peripheral serum VEGF level and its relavant factors in unstable angina pectoris patients. Chinese Journal of Geriatric Heart Brain Vessel Diseases. 2019;21:939-41.

26. Matusiak A, Chałubiński M, Broncel M, Rechciński T, Rudnicka K, Miszczyk E, et al. Putative consequences of exposure to Helicobacter pylori infection in patients with coronary heart disease in terms of humoral immune response and inflammation. Arch Med Sci. 2016;12:45-54.

27. Goukassian DA, Qin G, Dolan C, Murayama T, Silver M, Curry C, et al. Tumor necrosis factor-alpha receptor p75 is required in ischemia-induced neovascularization. Circulation. 2007;115:752-62.

28. Biasucci LM, Liuzzo G, Fantuzzi G, Caligiuri G, Rebuzzi AG, Ginnetti F, et al. Increasing levels of interleukin (IL)-1Ra and IL-6 during the first 2 days of hospitalization in unstable angina are associated with increased risk of in-hospital coronary events. Circulation. 1999;99:2079-84.

29. Sullivan GW, Sarembock IJ, Linden J. The role of inflammation in vascular diseases. J Leukoc Biol. 2000;67:591-602.

30. Waehre T, Halvorsen B, Damås JK, Yndestad A, Brosstad F, Gullestad L, et al. Inflammatory imbalance between IL-10 and TNFalpha in unstable angina potential plaque stabilizing effects of IL10. Eur J Clin Invest. 2002;32:803-10.

31. Tousoulis D, Antoniades C, Nikolopoulou A, Koniari K, Vasiliadou C, Marinou K, et al. Interaction between cytokines and SCD40L in patients with stable and unstable coronary syndromes. Eur J Clin Invest. 2007;37:623-8.

32. Huang D, Yang CZ, Yao L, Wang Y, Liao YH, Huang K. Activation and overexpression of PARP-1 in circulating mononuclear cells promote TNF-alpha and IL-6 expression in patients with unstable angina. Arch Med Res. 2008;39:775-84.

33. Abeyrathna P, Su Y. The critical role of Akt in cardiovascular function. Vascul Pharmacol. 2015;74:38-48. 
34. Pan Y, Yin J, Cai XM, Lin LI, Yi-Lan XU, Chun-Quan YU. Research progress on intervention of traditional Chinese medicine on coronary heart disease through PI3K/Akt signaling pathway. Chinese Traditional Herbal Drugs. 2017;48:4100-4.

35. Han J, Xuan JL, Hu HR, Chen ZW. [Protective effect against myocardial ischemia reperfusion injuries induced by hyperoside preconditioning and its relationship with PI3K/Akt signaling pathway in rats]. Zhongguo Zhong Yao Za Zhi. 2015;40:118-23.

36. Bennett MR, Evan GI, Schwartz SM. Apoptosis of rat vascular smooth muscle cells is regulated by p53-dependent and -independent pathways. Circ Res. 1995;77:266-73.

37. Bennett MR, Littlewood TD, Schwartz SM, Weissberg PL. Increased sensitivity of human vascular smooth muscle cells from atherosclerotic plaques to p53-mediated apoptosis. Circ Res. 1997;81:591-9.

38. Sheikh MS, Fornace AJ Jr. Role of p53 family members in apoptosis. J Cell Physiol. 2000;182:17181.

39. Li WTXDHEQYEXLJW. Changes in levels of serum visfatin and the relationships with activator protein-1 and MMP-2 in patients with angina pectoris. Shandong Medical Journal. 2016;56:1-3.

40. Zhao J, Li L, Peng L. MAPK1 up-regulates the expression of MALAT1 to promote the proliferation of cardiomyocytes through PI3K/AKT signaling pathway. Int J Clin Exp Pathol. 2015;8:15947-53.

41. Liu R, van Berlo JH, York AJ, Vagnozzi RJ, Maillet M, Molkentin JD. DUSP8 Regulates Cardiac Ventricular Remodeling by Altering ERK1/2 Signaling. Circ Res. 2016;119:249-60.

42. Indolfi C, Gasparri C, Vicinanza C, De Serio D, Boncompagni D, Mongiardo A, et al. Mitogen-activated protein kinases activation in T lymphocytes of patients with acute coronary syndromes. Basic Res Cardiol. 2011;106:667-79.

43. Eguchi S, Iwasaki H, Ueno H, Frank GD, Motley ED, Eguchi K, et al. Intracellular signaling of angiotensin II-induced p70 S6 kinase phosphorylation at Ser(411) in vascular smooth muscle cells. Possible requirement of epidermal growth factor receptor, Ras, extracellular signal-regulated kinase, and Akt. J Biol Chem. 1999;274:36843-51.

44. Kishimoto T, Taga T, Akira S. Cytokine signal transduction. Cell. 1994;76:253-62.

45. Shirogane T, Fukada T, Muller JM, Shima DT, Hibi M, Hirano T. Synergistic roles for Pim-1 and c-Myc in STAT3-mediated cell cycle progression and antiapoptosis. Immunity. 1999;11:709-19.

46. Minami M, Inoue M, Wei S, Takeda K, Matsumoto M, Kishimoto T, et al. STAT3 activation is a critical step in gp130-mediated terminal differentiation and growth arrest of a myeloid cell line. Proc Natl Acad Sci U S A. 1996;93:3963-6.

47. Catlett-Falcone R, Landowski TH, Oshiro MM, Turkson J, Levitzki A, Savino R, et al. Constitutive activation of Stat3 signaling confers resistance to apoptosis in human U266 myeloma cells. Immunity. 1999;10:105-15.

48. Rees ML, Subramaniam J, Li Y, Hamilton DJ, Frazier OH, Taegtmeyer H. A PKM2 signature in the failing heart. Biochem Biophys Res Commun. 2015;459:430-6. 
49. Cunha-Oliveira T, Ferreira LL, Coelho AR, Deus CM, Oliveira PJ. Doxorubicin triggers bioenergetic failure and p53 activation in mouse stem cell-derived cardiomyocytes. Toxicol Appl Pharmacol. 2018;348:1-13.

50. Yao K, Zhang WW, Yao L, Yang S, Nie W, Huang F. Carvedilol promotes mitochondrial biogenesis by regulating the PGC-1/TFAM pathway in human umbilical vein endothelial cells (HUVECs). Biochem Biophys Res Commun. 2016;470:961-6.

51. Sheeran FL, Pepe S. Mitochondrial Bioenergetics and Dysfunction in Failing Heart. Adv Exp Med Biol. 2017;982:65-80.

52. Karwi QG, Uddin GM, Ho KL, Lopaschuk GD. Loss of Metabolic Flexibility in the Failing Heart. Front Cardiovasc Med. 2018;5:68.

53. Ming DS, Hillhouse BJ, Guns ES, Eberding A, Xie S, Vimalanathan S, et al. Bioactive compounds from Rhodiola rosea (Crassulaceae). Phytother Res. 2005;19:740-3.

54. Zhang J, Liu A, Hou R, Zhang J, Jia X, Jiang W, et al. Salidroside protects cardiomyocyte against hypoxia-induced death: a HIF-1alpha-activated and VEGF-mediated pathway. Eur J Pharmacol. 2009;607:6-14.

55. Guan S, Feng H, Song B, Guo W, Xiong Y, Huang G, et al. Salidroside attenuates LPS-induced proinflammatory cytokine responses and improves survival in murine endotoxemia. Int Immunopharmacol. 2011;11:2194-9.

56. Xing S, Yang X, Li W, Bian F, Wu D, Chi J, et al. Salidroside stimulates mitochondrial biogenesis and protects against Hâñâ冈冈-induced endothelial dysfunction. Oxid Med Cell Longev. 2014;2014:904834.

57. Zhang JB, Guo JX, Cardiology DO. Protective effect of salidroside on human myocardial cells during hypoxia injury and possible mechanism. China Journal of Modern Medicine. 2017;27:21-6.

58. Kim S, Thiessen PA, Bolton EE, Chen J, Fu G, Gindulyte A, et al. PubChem Substance and Compound databases. Nucleic Acids Res. 2016;44:D1202-13.

59. Chai YQ, Zhao GH, Wang RJ, Cao MG, Wu HB, Tang SA, et al. [Anti-tumor metastatic constituents from Rhodiola wallichiana]. Zhongguo Zhong Yao Za Zhi. 2015;40:258-63.

60. Xue-jing W, Xue X, Xin L, Ya-ling S, Yi-wu Z, Wen-zhe H, et al. Chemical constituents from Rhodiola wallichiana var. cholaensis(â囚). Chinese Traditional Herbal Drugs. 2015;46:3471-4.

61. Wang XJLX, Zhou JMSYL, Zhao YWHWZ, Wang ZZXW. Chemical constituents from rhizomes of Rhodiola wallichiana var. issn.,2016.

\section{Tables}

Due to technical limitations, tables are only available as a download in the Supplemental Files section.

\section{Figures}




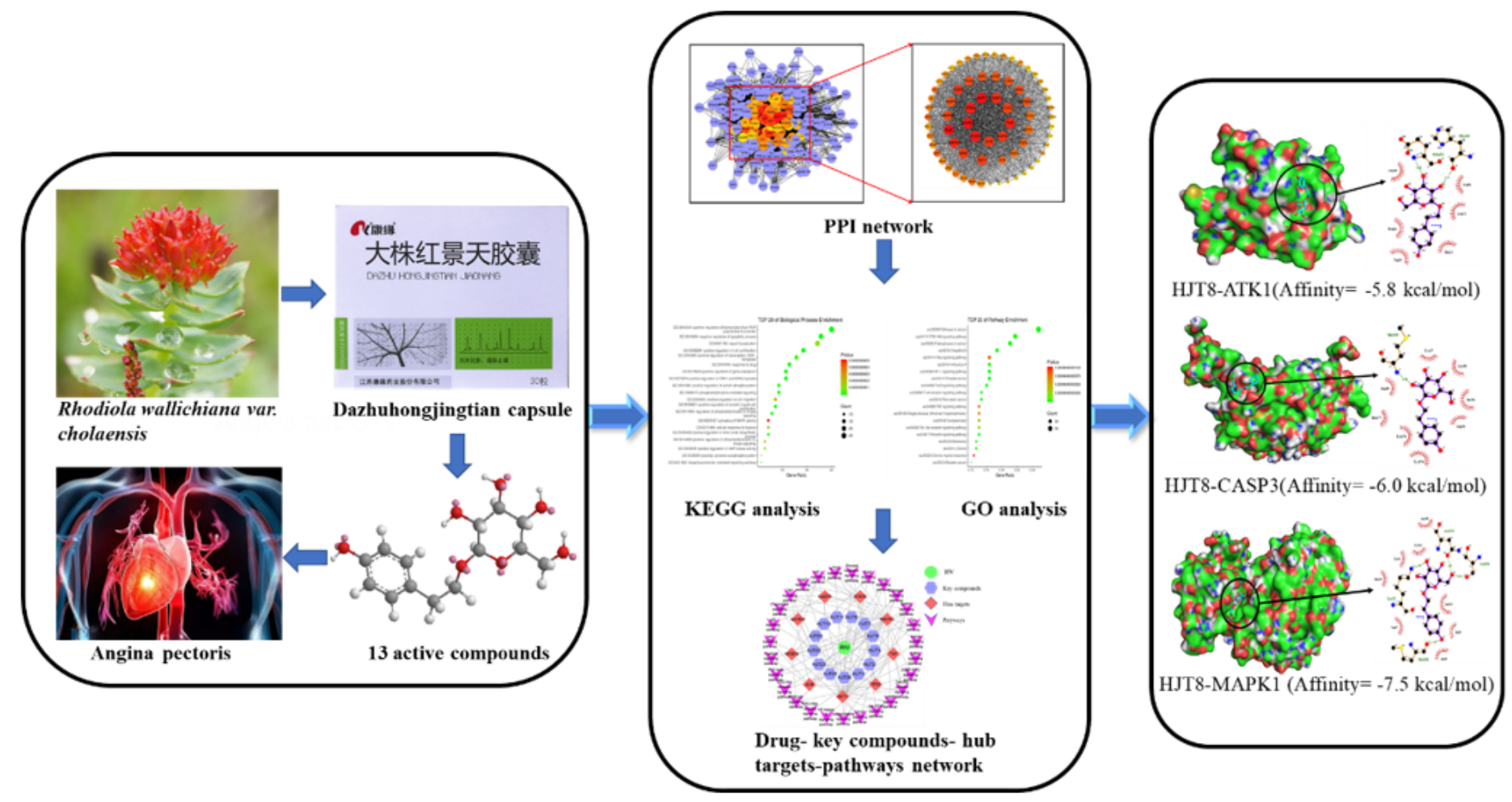

Figure 1

The pharmacological research flow chart of rhodiola in the treatment of angina pectoris (AP). 

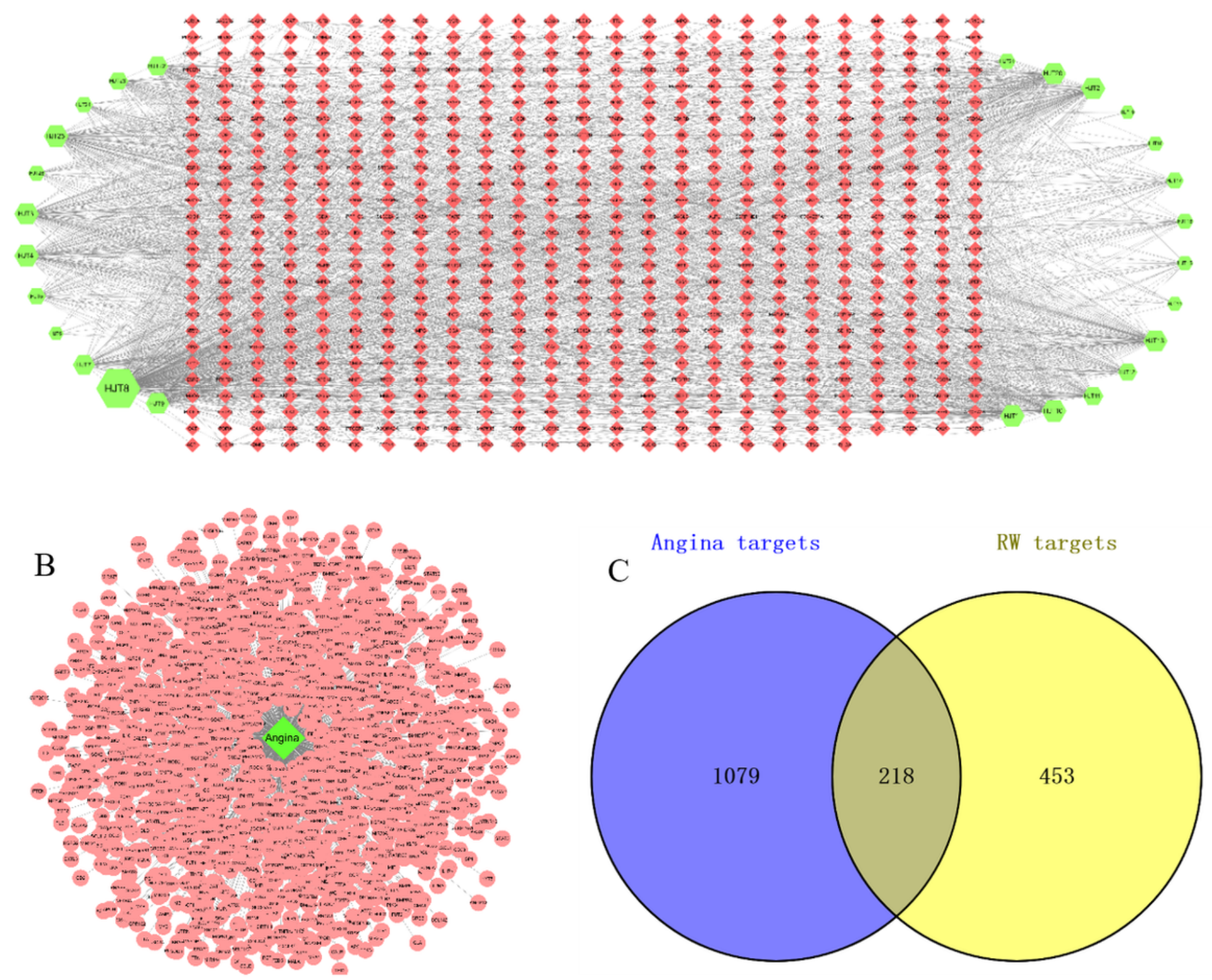

Figure 2

Analysis of the active compounds of RW and putative RW-angine. (A) Compound-predicted target network of RW. The green hexagon and red diamond represent RW compounds and RW targets. (B) Angina target network plotting. The green diamond and red circle represent disease and AP-targets. (C) Compound-RW/angina putative therapeutic target VennDiagram plot. The size of the nodes is directly proportional to the degree of the nodes. (The software of Cytoscape 3.7.2 was used to generate the figure). 


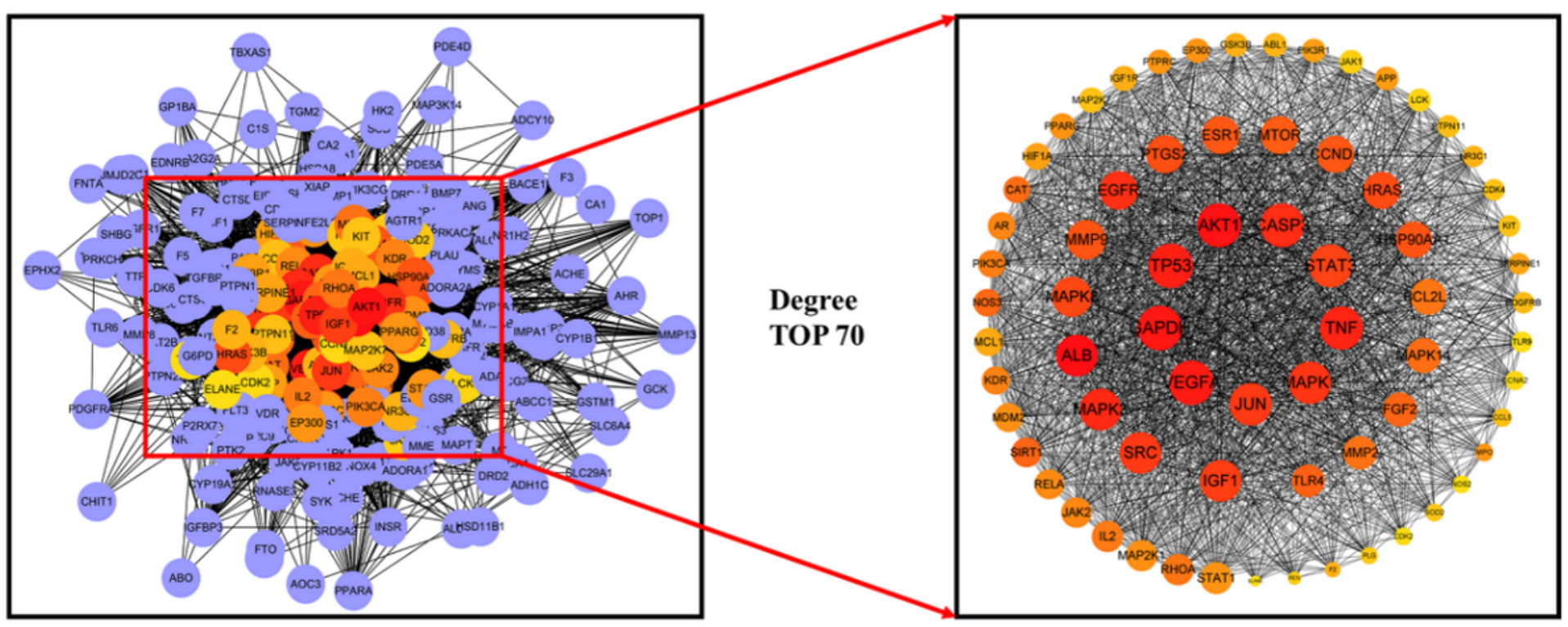

Figure 3

Identification of a core PPI network for RW against angina. (A) The interactive PPI network of baicalin and ischemic stroke targets comprising 218 nodes and 4456 edges is shown. (B) PPI network of significant proteins extracted from, this network comprises 70 nodes and 1689 edges.

$\mathbf{A}$

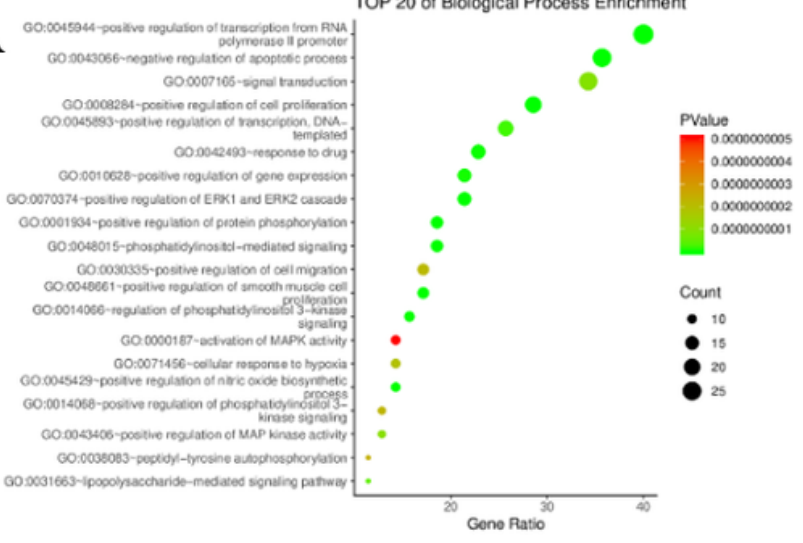

C

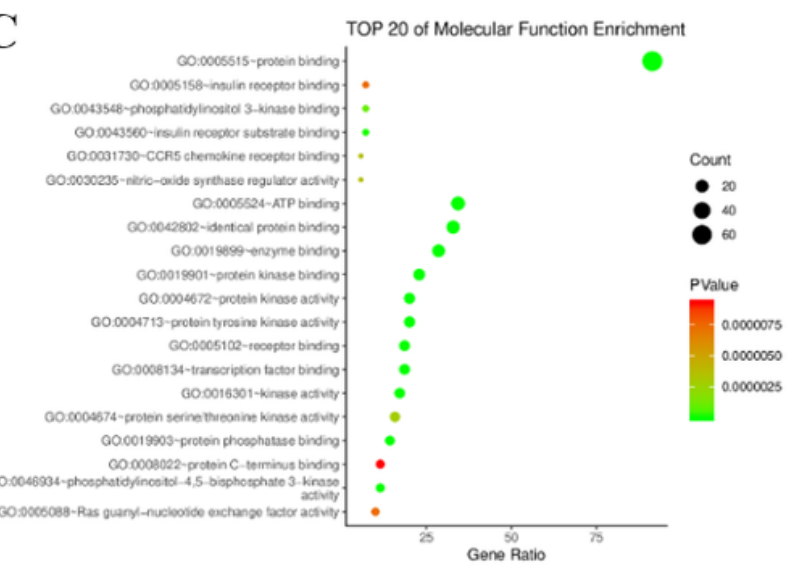

$\mathbf{B}$

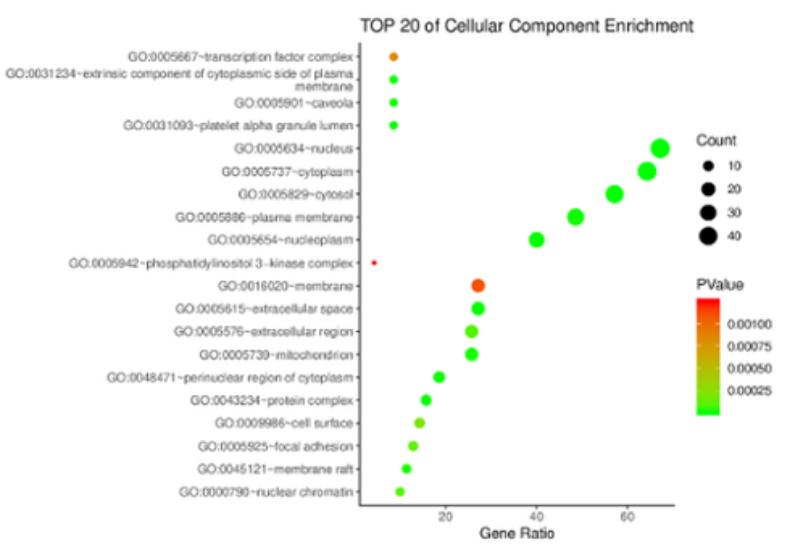

D

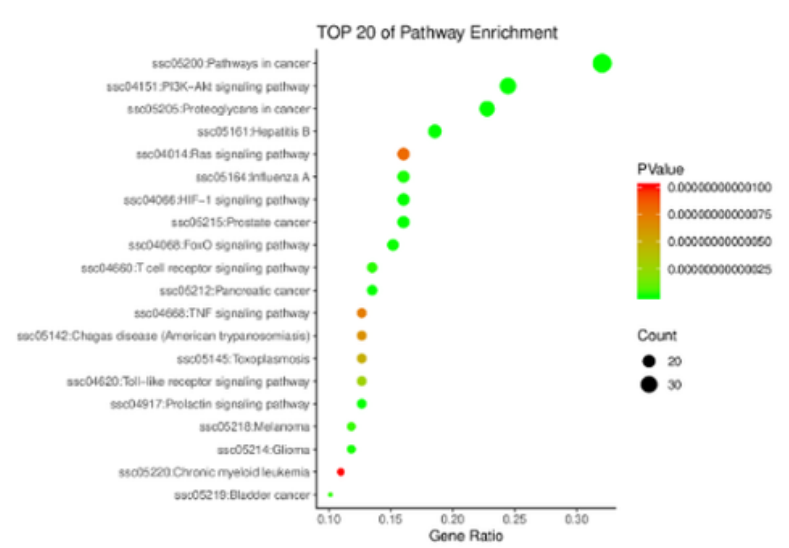




\section{Figure 4}

GO and KEGG Functional Annotation Analysis. (A) Enriched gene ontology terms for biological processes of potential targets; (B) Enriched gene ontology terms for cellular components of potential targets; (C) Enriched gene ontology terms for molecular functions of potential targets; (D) The enrichment analysis of the KEGG signaling pathways.

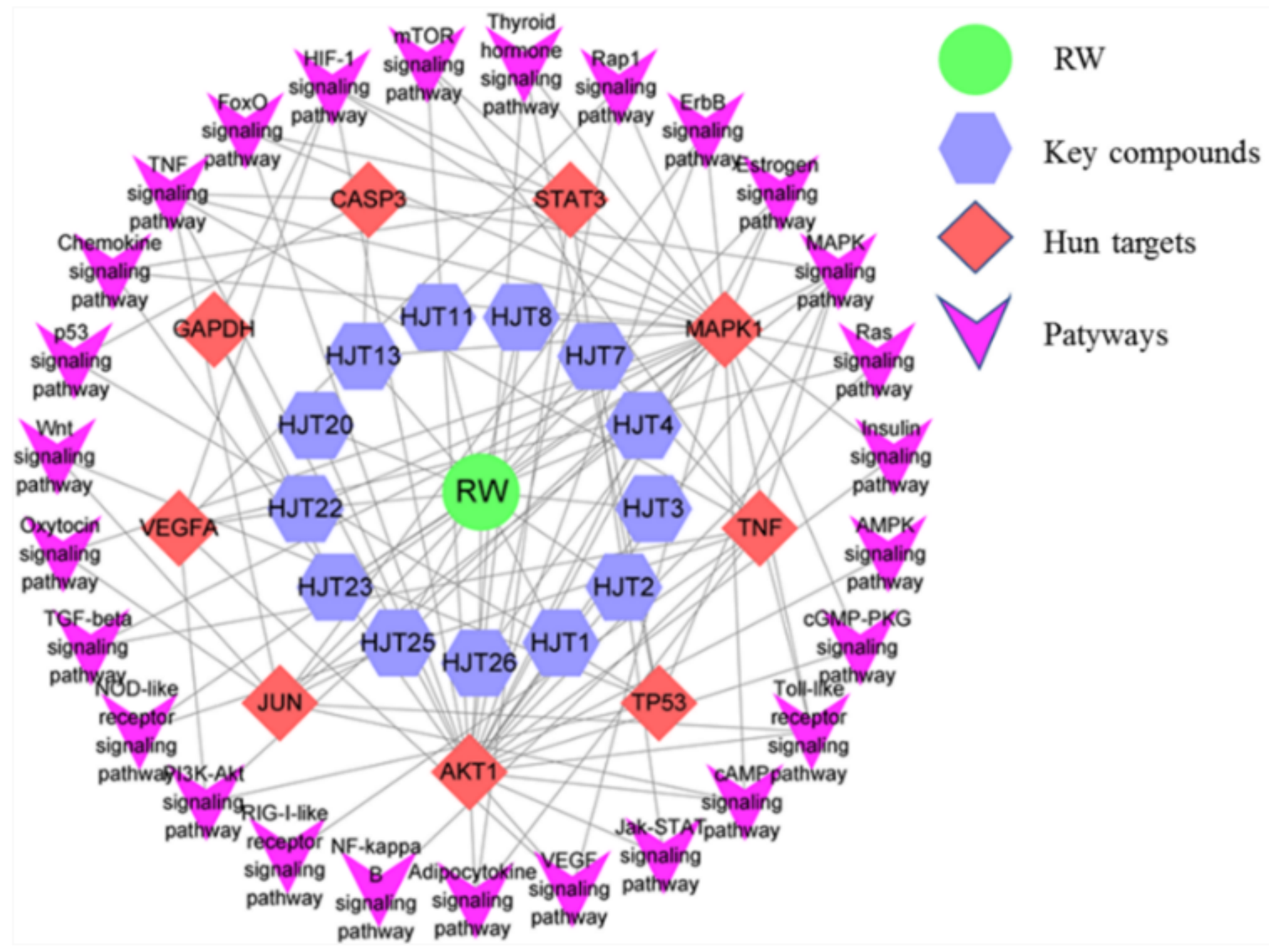

\section{Figure 5}

Drug-key compounds-hub targets-pathways network. The green circle represents drug, blue hexagon represents key compounds, red diamonds represent hub targets and purple arrow represents pathways. (The software of Cytoscape 3.7.2 was used to generate the figure). 


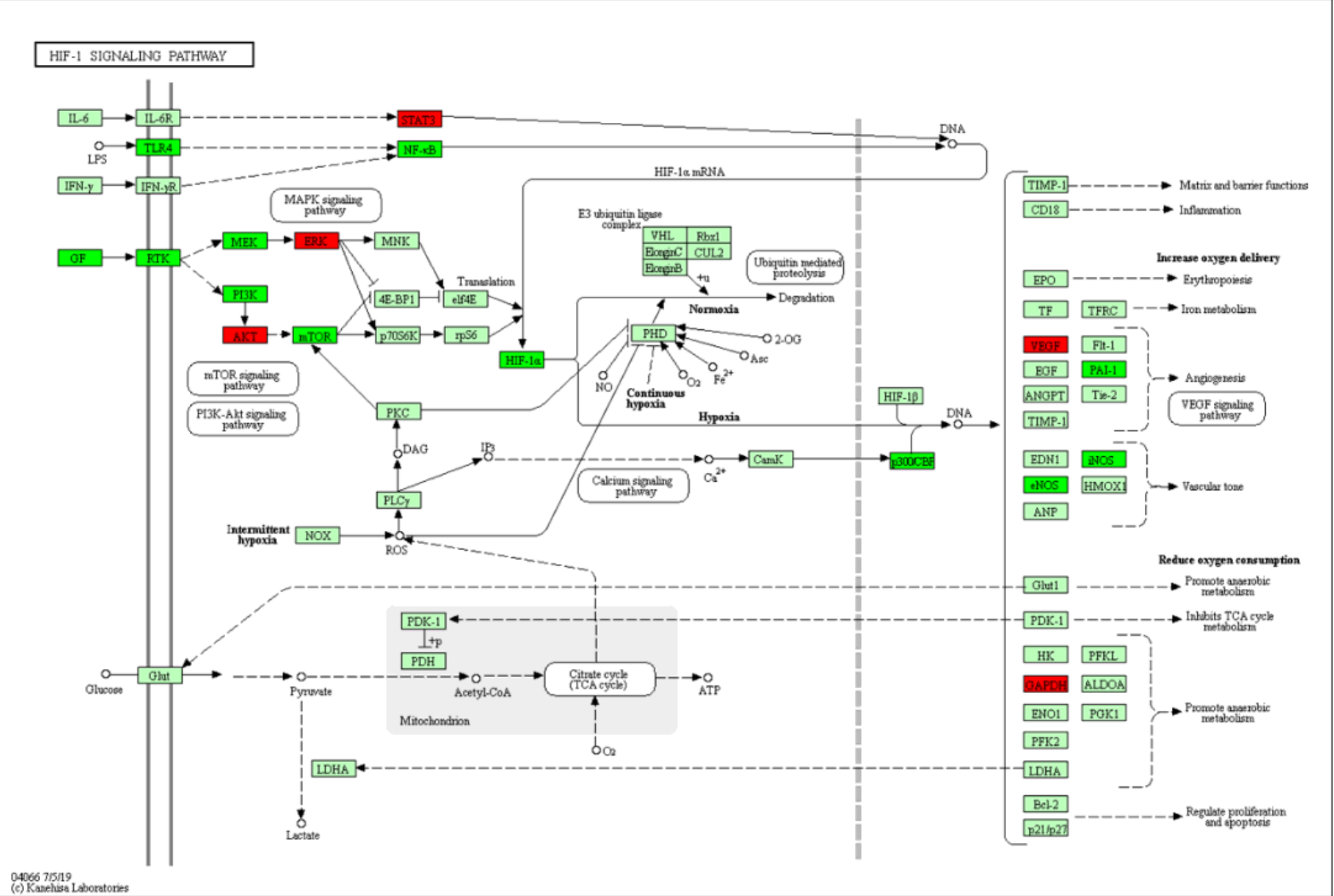

\section{Figure 6}

Modulating HIF-1 signaling pathway of RW against angina. Hub targets were colored in red, targets of $\mathrm{RW}$ - angina were colored in green.

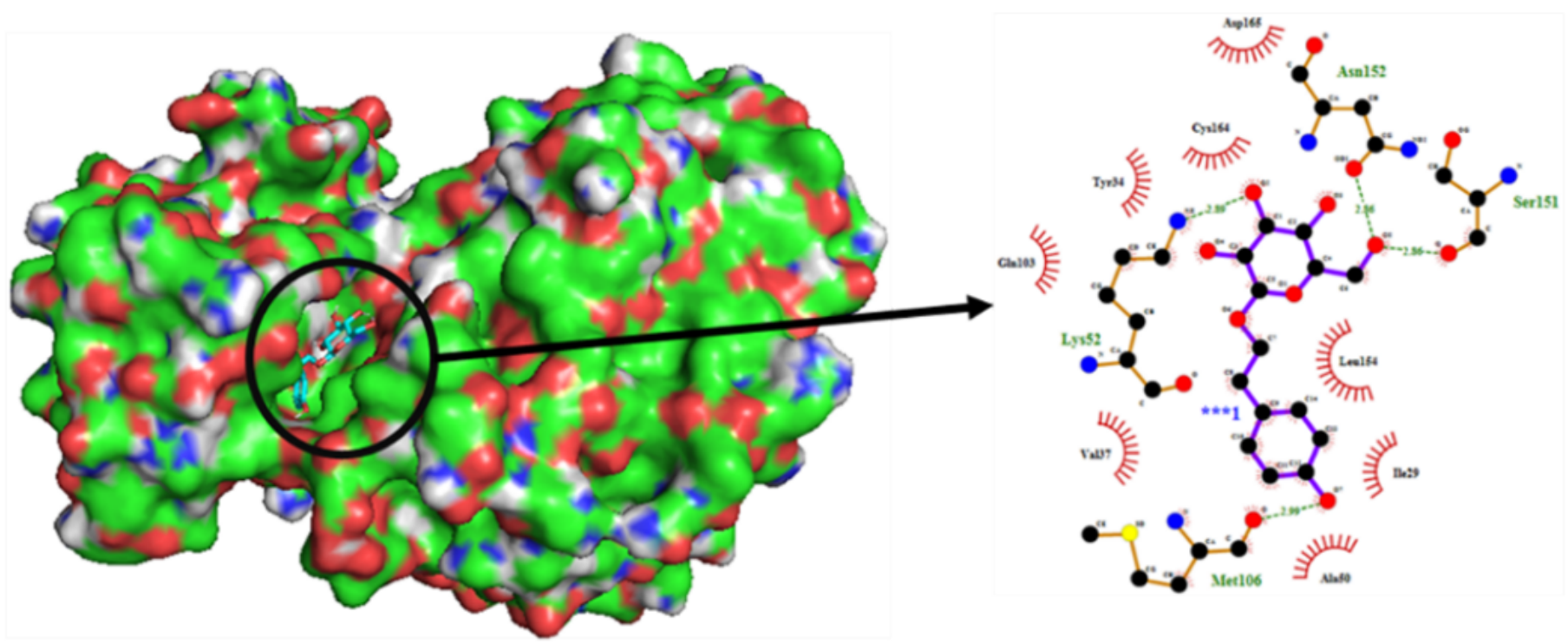

HJT8-MAPK1 (Affinity= $-7.5 \mathrm{kcal} / \mathrm{mol})$ 


\section{Figure 7}

Analysis of target-compound(HJT8) docking simulation. (The software of PyMOL 2.7 and LigPlot+ 1.4.5 were used to generate the figure).

\section{Supplementary Files}

This is a list of supplementary files associated with this preprint. Click to download.

- Table1Analysisofthe26underlyingcompoundsinRW.docx

- Table2Analysisoftarget.docx 